\title{
Contents, Vol. 117, 1949
}

\section{Index.}

Abt, K., und R. Bruckner: Netzhautgefäßspasmen bei artifiziell hypertonischen

Ratten 265

Scbl·ußwort: Abt. Amsler, M., et A. Huber: Dyshorie sympathique ...... 202

Discussion: Goldmann, Gut. Conclusion: Amsler. Amsler, M., et F. Verrey: Les injections de pénicilline et d'adrénaline dans la

chambre antérieure . . . . . . . . . . . . 243

Discussion: R. Bruckner, Goldmann, Saubermann. Appelmans, M., et H. van Vooren: Les

manifestations ophtaltnologiques de la

Myasthénie grave 8

Auerswald, W,: Beitrag zu den Unte $<$ rsuchungen über den Einfluß des «area

effects auf die Zeitabhängigkeit der Reizschwelle des dunkeladaptierten

Auges 104

Babel, J.: La surcharge graisseuse de la cornée

Balavoine, C, vide Franceschetti, A.

Bernoulli, R.: Beitrag zum Entstehungsmechandsinuis der Arteria hyak > idea

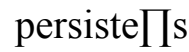
169

Blodi,F.: Naevus flammeus faciei und Glaukom ohne Vergrößerung des Bulbus 82 Blum, J. D.:

La tolerance des verres de contact dans la pratique; enquête auprès

de nos cinquante premiers cas 214

Discussion: Pascal. Böhringer, H. R.: Ectodermose pluriorificielle uind Auge .... 195

Diskussion: Babel. Brûcke, H. v., H. F. Hellauer und K. Umraith: Azetylcholin- und

Aneuringehalt

$\mathrm{d}^{1} / 8 \mathrm{r}$ Hornhaut und seine Beziehungen zur Nervenversorgung ... 19 Bruckner, R., vide Abt, K.

Busacca, Archimède: Sur la couleur verdâtre ou irisée du cysticerque intravitréen 347Cometta,

F.: Lymphome ou lymphosarcome de la conjonctive? . . . . 222

Cuendet, J. F.: Les variations saisonnières des affections oculaires .. $\quad .296$

Discussion: A. Bruckner, Goldmann. Conclusion: Cuendet.Cuendet, J. F., et V. Delia Porta: Une famille de nystagmiques . . . $\quad .199$

Discussion: Franceschetti. Conclusion: Cuendet.Dekking, H. M.: Toxoplasmosis as a Cause of

Congenital Defects ... 1

Delia Porta, V., vide Cuendet, J. F.

Doesschate, J. ten: Extra-Foveal S Scotopic Absolute Threshold and the Distribution of Retinal

Rods . .

.110Dufour, R.: Verres de contact en

matière plastique. Premiers résultats . 207Dufour, R., vide Schmitter, L.Esente, I.: Erste therapeutische Erfahrungen in der Behandlung des Glaukoms

mit der intravenösen Einspritzung von Para-aminobenzoyl-diäthyl-amino-

äthanol-chlorhydrat (Recorcaina, Novocain, Procain) .... 147 Etienne, R., vide Paufique, L.

Franceschetti, A., et C. Balavoine: LOpération prophylactique du décollement 
de la rétine

259

Franceschetti, A., et G. Maeder: Cataracte et affections cutanées dm type Poïkilodermie (Syndrome de Rothmund) et du type Sclérodermie (Syndrome de

Werner) 196

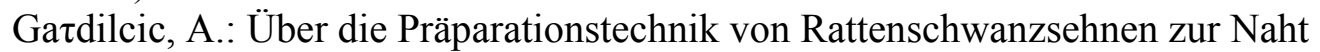

bei der Starausziebung

Gát, L.: Toxical Papilloretinitis as a Typical) Ocular Symptom in Tuberculotics 43

- Ulẅaseptyl--Einstreuuing in Eviszerationsstümpfe ..... 343Gig-er, Helen: Erfahrungen mil «Orthoptic-Training» ..... 325Goldmann, H.: Enthalten die Kammerwasservenen

Kammerwasser? . . . . 240

ScMußwoTt: Goldmann.

- $\quad$ Einige Ergebnisse der Spaltlampenuntersuchungen des Kammerwinkels und des Augenhintergrundles (hierzu Tafel II) .....

Hellauer, H. F., vide Brûcke, H. v. Huber, A.: Cerebrate und retinale Angiopathíe ...... 265

Diskussion: Goldmann, R. Bruckner. Huber, A., vide Amsler, M. Jokl, A.: Death from Eye

Disease and Occurrences of Death in Ophthalmological

Practice

Knüsel, O.: Weitere Beobachtungen an Kontaklschal·en .... 212

- $\quad$ Vitale Fãrbung in der Praxis 292

Knüsel, O.. Spätform der Cataracta eleetrica ...... 299

Diskussion: Strebel. Lisch, K.: i)ber familiäres Vorkommen von spontanem pulsierendem

Exophthal-

mus 180

Maeder, G., vide Franceschetti, A.

Moginier, A.: Implantation placentaire et acide nicotinique dans les rétinites

pigmentaires 287

Discussion: Pascal. Much, V.: Beitrag zur operativen Glaukom-Therapie ..... 36 Mailer, P.:

«Crocodil Chagrin» die la Membrane de Bowman à la suite d'une

Kératite traumatique avec hypopyon 225

Németh, B., vide Radnót, M.

Neuenschwander, M.: Unsere Erfahrungen mit der Placentatherapie

Oravisto, T.: On Ocular Symptoms in Arterial Hypertension .... 161

Pascal, J.I.: A Static and Dynamic Muscle Schema ..... 217

Discussion: Franceschetti, Strebel. Conclusion: Pascal. Paufique, L., et R. Etienne: Gliome et Pseudogiiome du Chiasma ... 90 Poleff, L.: Pathogénie allergique et microbienne des kératoconjonetivites

phlycténaires 338

Radnót, M., and B. Németh: On Which Eye Glaucoma Occurs More Frequently? 60 Rehsteiner, K.: Ein weiterer schweizerischer Stammbaum von dominant vererbter Retinitis pigmentosa

Roussel, F., vide Weekers, L.

Schmitter, L., et R. Dufour: Prothèses oculaires en plastique .... 293

Semadeni, B,. Tuberkulöse Panophthalmie ....... 273

Stein, A.: Beitrag zur Therapie des Ulcus serpens ..... 227

- $\quad$ Beitrag zur Therapie der Verätzungen ....... 232

Diskussion: A. Bruckner, Strebel, Haemmerli. Schlußwort: Stein. 
Umrath, K., vide Brücke, H. v.

Verrey, A.: La Loi Fédérale sur la circulation des véhicules à moteur et des cycles (15 mars 1932). Remarques à propos des conditions visuel·les . $\quad .195$

Verrey, F.: Reactions ten'sionnelles à la ponction de la chambre antérieure . 246

Discussion: Goldmann.

- $\quad$ Erfahrungen' mit der unbluligen Zyklodiatherm ${ }^{1 / 8}$ nach WeekeTs . .281Diskussion:

Haemmerli, R. Bruckner, Stein, Goldmann.

Verrey, F., vide Amsler, M.

Vooren, H. van, vide Appelmans, M.

Wagenaar, J. W.: On so-called Colour-Stereoscopy (With Plate I) .. $\quad .74$

Weber, E.: Einfaches Vorgehen bei intrakorneal abgebrochenen, tief in die

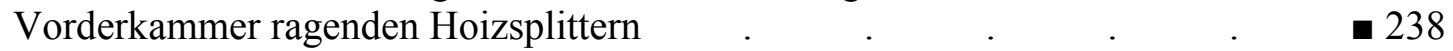

- $\quad$ Netzhautiöcher ohne Amotio ........ 263

Diskussion: Girardet.

Weekers, L., R. Weekers et F. Roussel: Mode d'action de la cyclodiathermie non

perforante 65

Weekers, L, et R. Weekers: Les fondements physiopathologiques et la technique

de Tiridencléisis 305

Weekers, L., vide Weekers, R. Weekers, R., vide Weekers, L. Witmer, R.: Bisherige

Erfahrungen bei der Entfernung nichtmagnetischer intra-

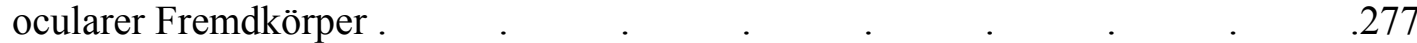

Diskussion: Koenig, Goldmann. Zollinger, R.: Über das Vorkommen von Gewebemastzellen in Iris undl Ziliar-

körper 249

GESELLSCHAFTSBERICHTE - SOCIETY TRANSACTIONS - SOCIËTÉS

Groupe Ophtalmologique du Léman. Reunion du 28 mars 1947 à Geneve . 186 Schweizerische Ophthalmologische Gesellschaft, 41. Generalversammlung in Zurich, 1.bis 3. Oktober 1948. Société Suisse dOphtalmologie, 41e as-semblée générale à Zurich, 1 au 3 octobre 1948 ..... 193 113th Meeting of the Netherlands Ophthalmological Society, held on the 13lh

a. and 14th of December

1947

in Utrecht 349

BUCHBESPRECHUNGEN - BOOKS REVIEW - LIVRES NOUVEAUX 63

127

300 